\title{
Smart Green Charging Scheme of Centralized Electric Vehicle Stations
}

Peter Makeen a,b,*, Saim Memon ${ }^{a}$, M. A. Elkasrawy ${ }^{c}$, Sameh O. Abdullatif $^{\mathrm{b}}$ and

Hani A. Ghali ${ }^{\mathrm{b}}$

${ }^{a}$ London Centre for Energy Engineering, Division of Electrical and Electronic Engineering,

School of Engineering, London South Bank University, UK; ${ }^{b}$ FabLab in the Center for Emerging Learning Technologies (CELT) and Electrical Engineering Department, Faculty of Engineering, The British University in Egypt (BUE), Cairo, Egypt; ${ }^{c}$ FabLab in the Center for Emerging Learning Technologies (CELT) and Renewable energy postgraduate programme, Faculty of Engineering, The British University in Egypt (BUE), Cairo, Egypt

*Correspondence: besadap@1sbu.ac.uk; 103 Borough Road, London, SE1 0AA, UK 


\section{Smart Green Charging Scheme of Centralized Electric Vehicle Stations}

Abstract: This paper presses a smart charging decision-making criterion that significantly contributes in enhancing the scheduling of the electric vehicles (EVs) during the charging process. The proposed criterion aims to optimize the charging time, select the charging methodology either DC constant current constant voltage (DC-CCCV) or DC multi-stage constant currents (DCMSCC), maximize the charging capacity as well as minimize the queuing delay per EV, especially during peak hours. The decision-making algorithms have been developed by utilizing metaheuristic algorithms including the Genetic Algorithm (GA) and Water Cycle Optimization Algorithm (WCOA). The utility of the proposed models has been investigated while considering the Mixed Integer Linear Programming (MILP) as a benchmark. Furthermore, the proposed models are seeded using the Monte Carlo simulation technique by estimating the EVs arriving density to the EVS across the day. WCOA has shown an overall reduction of $13 \%$ and $8.5 \%$ in the total charging time while referring to MILP and GA respectively.

Keywords: Electric Vehicles Station (EVS); PV-Grid integrated supply; Water Cycle Optimization technique (WCOT); Electric Vehicles (EV) charging time; Levelized Cost of Energy (LCOE)

\section{Introduction}

Recently, the projected size of the global electric vehicles (EVs) fleet is showing a booming trend, seeking a green sustainable eco-environment (Domínguez-Navarro et al. 2019; Amjad et al. 2018). Towards more sustainability, the integration of renewable energy sources in charging electrical vehicles is demonstrated in the literature (Domínguez-Navarro et al. 2019). Principally, EVs can be charged either in a centralized or decentralized manner (Amjad et al. 2018). For Decentralized charging, where AC is the only available choice, long charging time, overnight, is usually needed (Amjad et al. 2018). Consequently, researchers are tending to implement fastcharging centralized stations supplied from the utility grid and renewable energy resources to DC fast charge the EVs (Domínguez-Navarro et al. 2019).

For optimized charging performance in centralized stations, three case studies of feeding the electric vehicle stations (EVSs) based on the Monte Carlo method and GA have been applied using an objective function targeting maximum net present value (NPV) in (Domínguez-Navarro et al. 2019). The EVS is either supplied from the grid or the renewable energy sources or a mix between both of them. However, the contribution percentages of the renewable energy sources and 
the grid have not been stated in the third case. The optimal charging schedule of battery-swapping stations based on genetic and particle swarm optimization algorithms has been presented in $(\mathrm{Wu}$ et al. 2017). The targeted objective function has been considered as the electricity cost, the batteries used from the stock, and the potential damage due to various charging rates and implemented on four different battery charging scenarios: super, fast, normal, and slow chargers. In (Mehrjerdi and Hemmati 2020) the charging station has been supplied from the utility grid, wind energy system, and energy storage system based on stochastic mixed-integer programming. The operational sizing of the storage system and the charging time of EVs have been optimized. A mixed-integer nonlinear problem formulated the energy management system (EMS) of the utility grid with an EVS was introduced in (Ahmad et al. 2019). EMS is executed in balancing and forecasting the load demand with the generated power either from the utility grid or the PV or vehicle to vehicle (V2V) technology.-However, the utilization of simple linear programming techniques in the EV decision algorithm has not shown the expected robustness in capturing the global minimum of a given fitness function as reported in (Moghaddam et al. 2017; Ki et al. 2018). In (Huang and Kockelman 2020) genetic algorithm has been implemented to identify the profit-maximizing station placement and design in a congestion location. The model feedbacked the travelers by the optimum route choices under elastic demand and the charging price elasticity for the EV drivers. In (Chen et al. 2016) the authors proposed a framework for optimal routing and charging of the EVs based on minimizing the travel time and distance and maximizing the energy efficiency by reducing fuel and the charging cost. In (Korkas et al. 2017) approximate dynamic programming (ADP) method has been used to tackle a nearly-optimal control approach for charging and discharging of the EVs. The authors (Liang et al. 2020) presented joint charging scheduling, order dispatching, and EV rebalancing to a position.

Multi-charging options including AC charging, DC fast charging, and battery swapping has been presented in (Moghaddam et al. 2017). The decision-making options were implemented throughout the Ant Colony Optimization Algorithm (ACOA). The algorithm targeted the reduction of the travel time, the charging time, and the charging cost. A queue mapping of EVs based on the Markov decision process (MDP) was declared in (Zhang et al. 2013). Optimal scheduling was achieved based on the EV arrival time, the intermittency of renewable energy, the grid power price, and the charging energy of the EVs (Zhang et al. 2013). In (Ucer et al. 2019) DC-fast charging station based on the constant current-constant voltage method has been presented using Monte 
Carlo analysis to quantify the effect of EV loading on the station. However, the charge acceptance curves of the EV should be implemented before the charging process. The charge acceptance curves used to limit the power used in charging the EV and have been categorized into three main categories (24 kWh, $60 \mathrm{kWh}$, and $85 \mathrm{kWh}$ ). In (Said and Mouftah 2019), the Peak-load management (PLM) scheme has been used to schedule EVs for charging and discharging. The EV supply equipment selection model has been investigated to guide EVs to the best supply station corresponding to the power demand, timing, and location. In (Zhang, You, and Cai 2018) the number of EVs that leave the EVS without charging has been minimized, with an optimal pricing approach has been applied in the station. Long term profit of EVS owner and minimizing delay time of EVs have been discussed in (Rabiee, Ghiasian, and Chermahini 2018). EVS owner has the control to set the charging process of queues. The Joint Admission and Pricing (JoAP) mechanism has been applied in (Wang et al. 2018). JoAP optimized the admission rate control, electricity price, and EVs arrival rate. However, the impact of the efficiency of the charging station including integration between renewable energy sources and distributed energy generations were not declared. While various attempts have been introduced in the literature to develop an EVS decision-making algorithm, a limitation in considering a constant partial charging capacity was always reported with a limited EV database as well. In (Gupta, Rana, and Mishra 2020) a scheduling method of the EV users while traveling on a highway has been proposed based on the priority selection using their state of charge $(\mathrm{SoC})$ data. An orderly charging scheduling algorithm based on user bidding has been declared in (Cheng et al. 2020). The charging priority has been determined according to the user bid and the charging status of the EV.

This paper presses novel smart green charging decision-making algorithms to be utilized in the electric vehicle charging stations. The estimated number of EVs entering the station is simulated using the sequential Monte Carlo simulation technique. The developed criterion is capable of scheduling the EVs entering the centralized station through a fitness function with variable weighting factors. The novelty of the proposed centralized approach is based on minimizing the total interval charging time of the entire station, determining the targeted partial charging capacity, and classifying the charging methodology for each EV. The effectiveness of the metaheuristic algorithms has been addressed with respect to linear programming showing the proposed model superiority. 


\section{The system under Feasibility Study}

The proposed EVS, under investigation, is chosen to be localized in El-Sherouk City, Egypt $(30,07,03, \mathrm{~N}-31,36,20, \mathrm{E})$. EVS under feasibility consists of a photovoltaic (PV) system with a maximum power point tracking system (MPPT), Batteries, Utility grid, DC-AC inverter, net metering, and DC-DC converter to charge electric vehicles as shown in Fig. 1. The EVS composes of ten DC charging points which can be used for DC-constant current constant voltage (DCCCCV) or DC-multi-stage charging current (DC-MSCC) charging methodologies and powered only from the PV system in the morning while in the evening the station pumped from the grid. The total energy consumed of the proposed electric vehicle station has been assumed during the day, based on the study in (Reddi et al. 2016), as presented in Fig. 2.

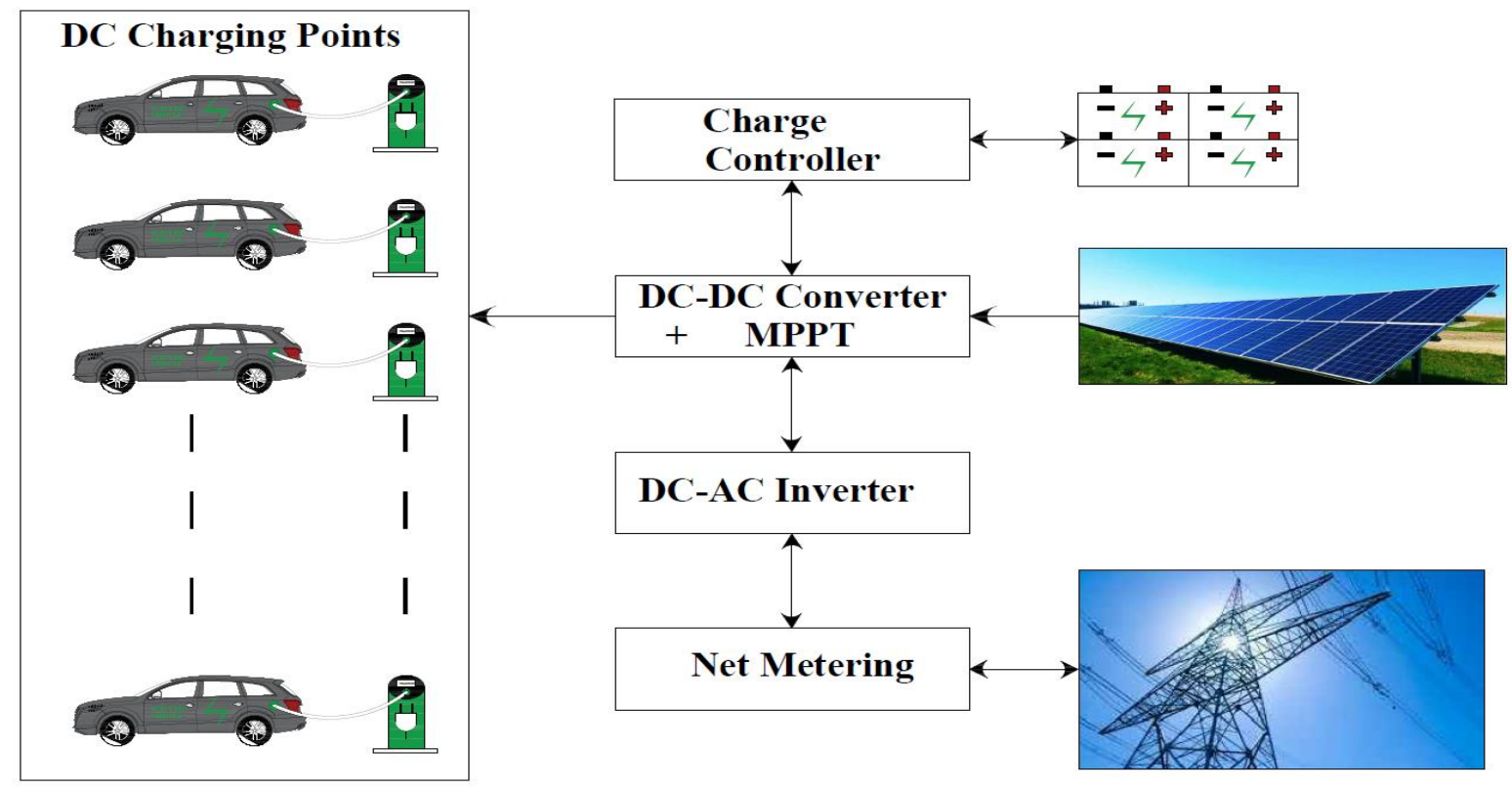

Figure 1. A schematic for the proposed electric vehicle station localized in El-Sherouk City, Egypt. 


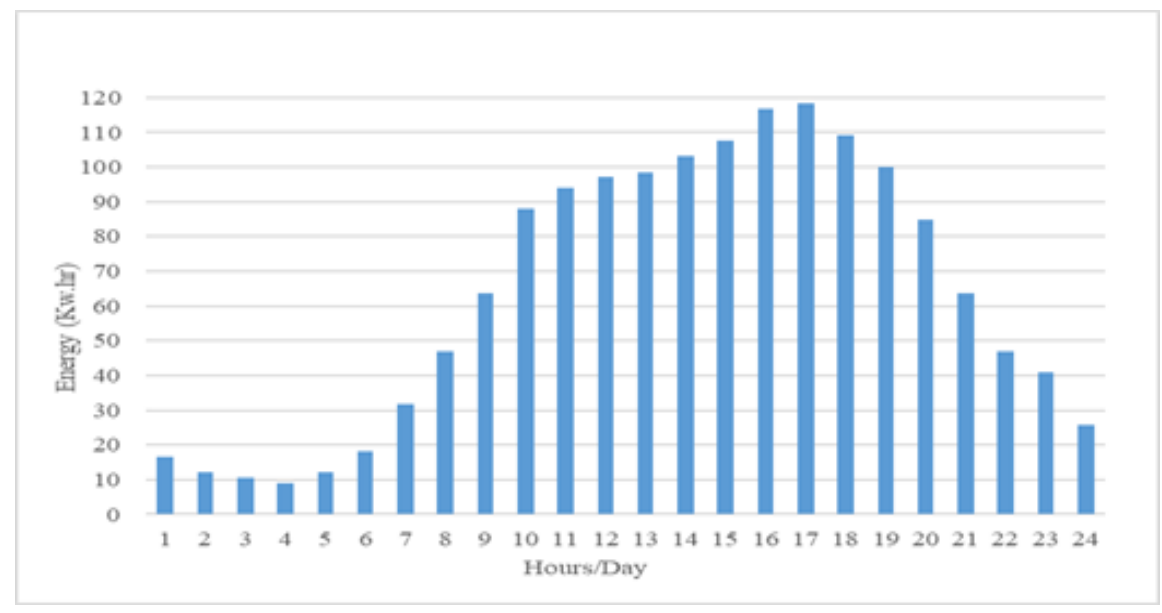

Figure 2. Energy consumed by the proposed electric vehicle station during the day

In this paper, the number of EVs entering the EVS has been analyzed through the sequential Monte Carlo simulation technique (Domínguez-Navarro et al. 2019; Vorobjovs, Berzma, and Zirovecka 2018). This technique presents the flow of the electric vehicles that arrive at the station with a finite number of charging points (ten points). The sequential Monte Carlo simulation technique has been iterated for ten successive runs where the average number of EVs has been observed to be $15 \mathrm{EVs}$ at peak working hour $(5: 00 \mathrm{pm})$ as shown in Fig. 3. The estimated number of vehicles at peak hour is consistent with corresponding systems in literature as in (Ucer et al. 2019).

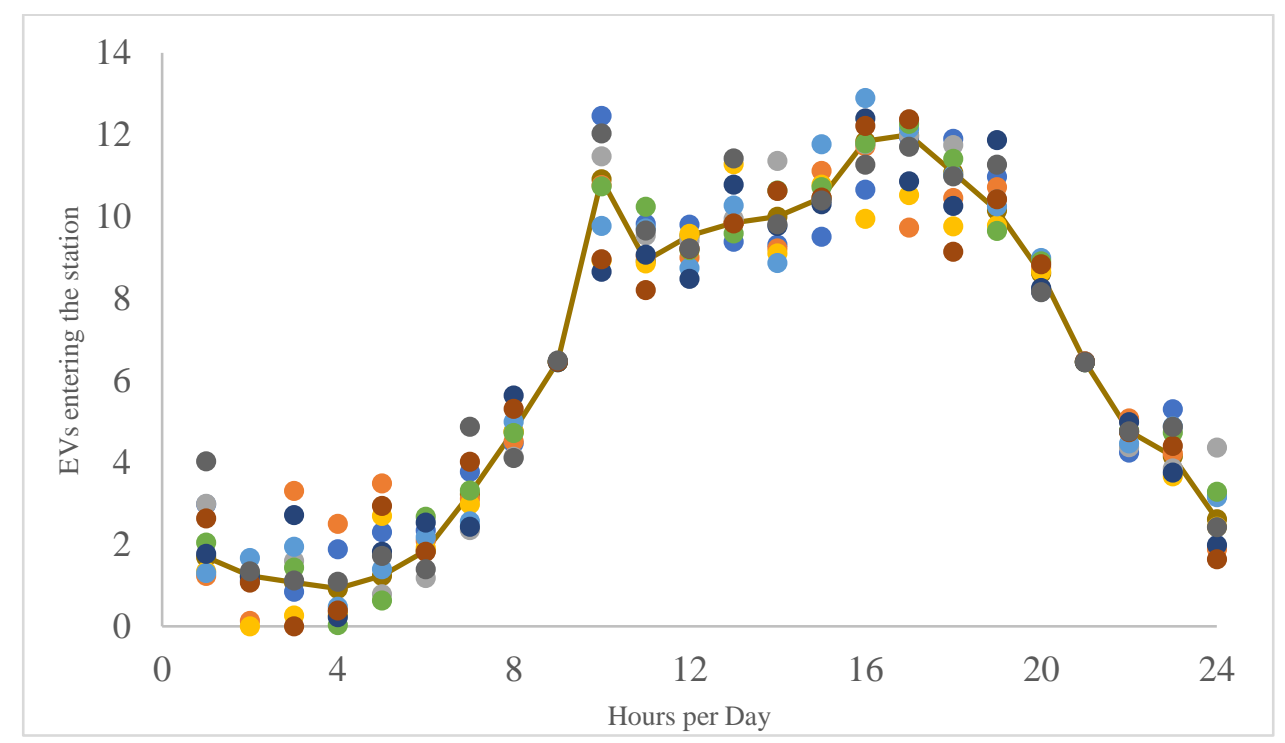

Figure 3. The Monte Carlo technique is used to predict the average number of EVs entering the EVS across the day, samples of ten successive runs are plotted. 
To study the feasibility of the proposed system, 15 various cars are used to emulate the different charging scenarios in the station with a randomly chosen initial state of charge per each, while the energy required for full capacity has been estimated using each EV full capacity, initial SOC, and voltage (c.f. Table 1). The DC-CCCV charging time has been captured from the datasheet of each corresponding EV. However, the DC-MSCC charging time has been estimated by the multi-stage charging current methodology based on Cuckoo Optimization Algorithm (COA) implemented in (Makeen, Ghali, and Memon 2020b; Makeen, Ghali, and Memon 2020a).

TABLE 1. Proposed EVs with various charging methodologies (https://evdatabase.org/car/1178/SEAT-el-Born\#charge-table Last accsssed: 18/04/2020)

\begin{tabular}{cccccc}
\hline \hline & & \multicolumn{3}{c}{ Charging Methodology } & Remaini \\
Car_\# & Car type & $\begin{array}{c}\text { DC-CCCV } \\
(\mathrm{min})\end{array}$ & $\begin{array}{c}\text { DC-MSCC } \\
(\mathrm{min})\end{array}$ & $\begin{array}{c}\text { Energy } \\
(\mathrm{kWh})\end{array}$ & $\begin{array}{c}\text { ng SOC } \\
(\%)\end{array}$ \\
\hline Car_1 & Mercedes EQC 400 (4 & 30 & 23.265 & 80 & 20 \\
& matic) & & & & \\
Car_2 & CHEVROLET SPARK & 20 & 15.51 & 19 & 15 \\
Car_3 & Ford Focus (2013) & 30 & 23.265 & 23 & 16 \\
Car_4 & Fiat 500 (2018) & 30 & 23.265 & 24 & 18 \\
Car_5 & Nissan leaf (2016) & 30 & 23.265 & 24 & 21 \\
Car_6 & E-Golf (2018) & 40 & 31.02 & 24 & 10 \\
Car_7 & SEAT-el-Born (2019) & 30 & 23.265 & 62 & 8 \\
Car_8 & Mitsubishi I-Mi (2012) & 20 & 15.51 & 16 & 23 \\
Car_9 & Kia Soul (2015) & 33 & 25.592 & 27 & 25 \\
Car_10 & BMW i3 (2014) & 30 & 23.265 & 18.8 & 9 \\
Car_11 & Toyota RAv2 (2014) & 30 & 23.265 & 8.8 & 12 \\
Car_12 & Honda Clarity (2020) & 35 & 27.143 & 17 & 11 \\
Car_13 & Mercedes EQC 400 (4 & 30 & 23.265 & 80 & 9 \\
& matic) & & & & \\
Car_14 & CHEVROLET SPARK & 20 & 15.51 & 19 & 10 \\
& (2016) & & & &
\end{tabular}




\begin{tabular}{llllll} 
Car_15 & Ford Focus (2013) & 30 & 23.265 & 23 & 8 \\
\hline \hline
\end{tabular}

\section{Electrical Vehicle Station Smart Charging Strategy}

\section{Levelized Cost of Energy (LCOE)}

In general, to start calculating the LCOE, PV sizing calculations of the energy demand in $\mathrm{kWh}$ presented by the load profile in Fig. 2, have to be determined and multiplied by a safety factor from $20 \%$ to $30 \%$ depending upon the topology of the system, either a stand-alone or gridconnected to compensate the systems losses. As a first step, the overall PV system capacity is determined based on the peak sun hours of the desired location, an average of 5.8 hours is used in the case of Egypt. Consequently, the number of panels was calculated using:

$$
\mathrm{N}_{\mathrm{P}}=\frac{\mathrm{E}_{\mathrm{L}} \times(1+\text { losses \% })}{\mathrm{P}_{\mathrm{P}} \times \mathrm{PSH}}
$$

where $N_{P}$ is the number of required panels, $E_{L}$ is the load energy consumption (Wh/day), Losses $\%$ is the overall system losses, $\mathrm{P}_{\mathrm{P}}$ is the panel power (W) and PSH is peak sun hour (h/day).

Following that, the needed number of inverters/converters is calculated in terms of the overall system power as well as the rated power per selected inverter/converter using:

$$
\mathrm{N}_{\text {inv }}=\frac{\left(\mathrm{E}_{\mathrm{L}} \times(1+\text { losses \% }) / \mathrm{T}_{\mathrm{w}}\right)}{\mathrm{P}_{\mathrm{inv}}}
$$

where, $N_{\text {inv }}$ is the number of inverters/converters, $P_{\text {inv }}$ rated power per each inverter $(k W), T_{w}$ is the charging time in $\mathrm{h} /$ day.

Finally, the charging bank of storage batteries is estimated for the fourth scenario where a fully standalone system is proposed by:

$$
\mathrm{E}_{\mathrm{L}} \times(1+\text { losses \% }) \times(\mathrm{N}+1) \times \frac{1}{\mathrm{DOD}} \times \frac{1}{\eta_{\mathrm{B}}} \times \frac{1}{\mathrm{~V}_{\mathrm{B}}} \times \frac{1}{\mathrm{Ah}}=\mathrm{N}_{\mathrm{B}}
$$

where $N_{B}$ is the number of storage batteries, DOD is the depth of discharge, $\eta_{B}$ is battery efficiency, $A h$ is the battery ampere-hour and $V_{B}$ is battery voltage. In order to evaluate the amount of excess energy pumped to the grid during the morning time, the following equation is involved:

$$
E_{E}=\frac{\left(E_{P}\right)-\left(E_{C}\right)}{\left(E_{P}\right)} \times 100
$$


where, $E_{E}$ is the percentage of excess energy per year, $\left(E_{P}\right)$ is the energy supplied per year and $\left(E_{C}\right)$ is the energy consumption per year. The PV system specifications are listed in Table 2.

TABLE 2. The PV system specifications

\begin{tabular}{|c|c|}
\hline Project location Cairo assuming (PSH) & $5.8 \mathrm{~h}$ \\
\hline PV Panel specification & $0.335 \mathrm{~kW}$ \\
\hline Length & $1.988 \mathrm{~m}$ \\
\hline Width & $0.992 \mathrm{~m}$ \\
\hline Vmp & $34.7 \mathrm{~V}$ \\
\hline $\operatorname{Imp}$ & $7.12 \mathrm{~A}$ \\
\hline Clearance shadowing area & $0.3 \%$ \\
\hline Price & $5450 \mathrm{LE} / \mathrm{kW}$ \\
\hline Inverter specification for PV off-grid load $(5 \mathrm{~kW})$ & $13965 \mathrm{LE}$ \\
\hline Structure price & $1700 \mathrm{LE} / \mathrm{kW}$ \\
\hline Connection price & $810 \mathrm{LE} / \mathrm{kW}$ \\
\hline Battery no of charging cycle & 4000 Cycles \\
\hline \multirow{4}{*}{ Battery specification (12 v/ $200 \mathrm{AH})$} & $200 \mathrm{Ah}$ \\
\hline & $12 \mathrm{~V}$ \\
\hline & $0.2 \mathrm{DOD} \%$ \\
\hline & 4764.42 LE \\
\hline Number of cloudy days & 2.5 \\
\hline Total energy consumption per day & $\begin{array}{c}1244.880274 \\
\text { kW/day }\end{array}$ \\
\hline Total solar PV power & $214.6345299 \mathrm{~kW}$ \\
\hline No. of PV panels used & 641 \\
\hline Area of the system & $1643.347597 \mathrm{~m}^{2}$ \\
\hline No. of inverters & 7 \\
\hline Capital cost & $2208290.858 \mathrm{LE}$ \\
\hline Capital cost for Morning DC load & $1708490.858 \mathrm{LE}$ \\
\hline
\end{tabular}


To engage the economic side in the current study, the Levelized Cost of Energy (LCOE) for the different scenarios $(\varepsilon)$ has been calculated by:

$$
L C O E=\frac{\mathrm{C}_{\mathrm{C}}+\sum\left(\mathrm{R}_{\mathrm{C}}-\mathrm{E}_{\mathrm{S}}\right)}{\sum \mathrm{E}_{\mathrm{P}}}
$$

where $C_{C}$ is the capital cost $(\mathrm{LE}), \mathrm{R}_{\mathrm{C}}$ is the Running cost and $\mathrm{E}_{\mathrm{S}}$ is the Energy sold to the grid in (LE).

The proposed EVS operates as the DC charging points in the morning will be powered only from the PV system while in the evening the station will be pumped from the grid through the AC/DC converter. The LCOE for the DC-CCCV charging methodology during the morning and evening is assumed to be $0.75 \mathrm{LE}$ and $4.84 \mathrm{LE}$ respectively. Furthermore, the LCOE of the DCMSCC charging methodology used in this paper has been estimated to be $20 \%$ higher than the DCCCCV methodology due to the controller used to acquire the multi-stage charging currents with their interval times based on the COA initial introduced in our previous work in (Makeen, Ghali, and Memon 2020b).

\section{Charging Capacity $\left(\boldsymbol{C}_{c h}\right)$}

The desired charging capacity for each EV is depending on the charging cost of each charging methodology and its current SOC As given in:

$$
C_{c h_{\lambda}}=L C O E \times\left(\delta-S O C_{\lambda}\right) \times \mathrm{E}
$$

Where $\delta$ is the coefficient of partial charging capacity which can vary from the SOC of current state to a range of targeted SOC varies from $85 \%$ to $100 \%$, and $\mathrm{E}$ is the total capacity of the corresponding EV.

\section{Total Charging Time $\left(T_{T}\right)$}

Each EV has a different total charging time in the EVS due to the variable charging alternative DC-CCCV or DC-MSCC and the queuing time to reach the charging point. The total charging time for each EV in the EVS can be expressed as

$$
T_{T_{\lambda}}=T_{P_{\lambda}}+T_{C h_{\lambda}}+T_{W_{\lambda}}
$$


Where $T_{P_{\lambda}}$ is the parking time of each EV in the station and has been assumed to be constant of 3 mins, $T_{C h_{\lambda}}$ is the charging time of each EV according to the charging option DC-CCCV or DCMSCC and $T_{W_{\lambda}}$ is the total waiting time in the queue to be charged from the charging point.

\section{Decision Making Criteria}

In this section, a decision-making criterion is applied to optimize the total charging time corresponding to the charging methodology and the optimum coefficient of partial charging capacity of each EV based on a comparison between the Mixed Integer Linear Programming (MILP) and metaheuristic algorithms, including Genetic Algorithm (GA) and Water Cycle Optimization Algorithm (WCOA). The coming two subsections demonstrate the proposed EVS decision-making algorithm based on MILP and metaheuristic algorithms respectively.

\section{Mixed Integer Linear Programming (MILP)}

MILP is considered as a mathematical optimization process with a condition that all the variables are integers (Trespalacios and Grossmann 2014). The optimum charging methodology either DCCCCV or DC-MSCC and overall charging time corresponding to the maximum preferable capacity corresponding to the current SOC of each EVs have been integrated as a linear equation as follow

$$
\begin{gathered}
\zeta_{\left.\lambda\right|_{D C-C C C V,}}=\frac{\beta_{1} \rho_{\text {nor } \cdot \lambda}+\beta_{2}\left(T_{T_{\lambda}}\right)_{n o r \cdot \lambda}+\beta_{3}\left(C_{c h_{\lambda}}\right)_{n o r \cdot \lambda}}{3} \\
\sum_{i=1}^{3} \beta_{i}=3, \quad \lambda=1,2, \ldots \ldots, 15
\end{gathered}
$$

Where $\lambda$ is an incremental counter for the EVs entering the station at the same time and it varies from 1 to $15, \beta$ is the weighting factor of the corresponding elements and $\rho$ is the $\mathrm{EV}$ arriving density. Herein, all parameters have been normalized with respect to the maximum as indicated by the subscript nor.

\section{Metaheuristic Algorithms}

In the previous section, the proposed smart charging scheme has been formulated as a classical optimization problem. However, the optimization solution is hard to be implemented through linear programming, so metaheuristic algorithms are recommended to solve such research 
problems (Moghaddam et al. 2017; Ki et al. 2018). Various algorithms are used to solve optimization problems, which guarantee to obtain the global optimal solution for the studied system. Recently, researchers tend to use a meta-heuristic algorithm based on natural inspiration. The algorithms combine rules and randomness of the natural phenomena (Shi et al. 2016; Makeen et al. 2018).

The MILP which is presented in eq. (8) has been updated to be more precise in ranking the EVs and ensure the optimum solution of fast charging the EV with its corresponding current and targeted SOC as

$$
\underset{D C-M S C C}{\left.\zeta_{\lambda}\right|_{D C-C C C V}=\sqrt[3]{\rho^{\beta_{1}} \times\left(T_{T_{\lambda}}\right)_{\text {nor } \cdot \lambda}^{\beta_{2}} \times\left(C_{C h_{\lambda}}\right)_{\text {nor } \cdot \lambda}^{\beta_{3}}}}
$$

The proposed fitness function is complex in nature and quite difficult to solve because there is more than one local optimum in the problem and multiple constraints have to be satisfied. Although the gradient search may be unstable for solving such problems to avoid getting trapped in local optima. So, in this paper two metaheuristic algorithms, GA and WCOA have been implemented to have the optimum charging scheme efficiency, accuracy, overall charging time as well as queuing delay of the EVs. The results of the metaheuristic algorithms have been compared with each other and with the MILP. The proposed MILP and metaheuristic algorithms (GA and WCOA) are targeting to minimize the objective functions in eq. (8) and eq. (10) respectively. The decision-making criteria are based on the random selection of the parameters within the permitted constraints.

GA is considered as the reference of the metaheuristic algorithms as it is inspired by the biological evolution process of computational data and the mechanism of natural genetics selection (Abdel-aal 2012; Dasgupta and Michalewicz 2013). GA is composed of the initial population, fitness evaluation function, reproduction approach, crossover operator, and mutation operator. These operators result in an optimum solution using a fitness function, as it maps the natural objective function and the results have been compared with the WCOA.

WCOA is inspired by the nature of the water cycle based on the flow of the rivers and streams toward the sea. This algorithm ensured more precise performance compared with other alternating algorithms, this can be attributed to its efficiency, accuracy in terms of the number of 
function evaluations (Eskandar et al. 2012; Shi et al. 2016). The deployment of WCOT consisting of the creation of the initial population, flowing of streams to the rivers and sea, the evaporation process, the raining process, and the constraints of the algorithm have been demonstrated in Fig. 4.

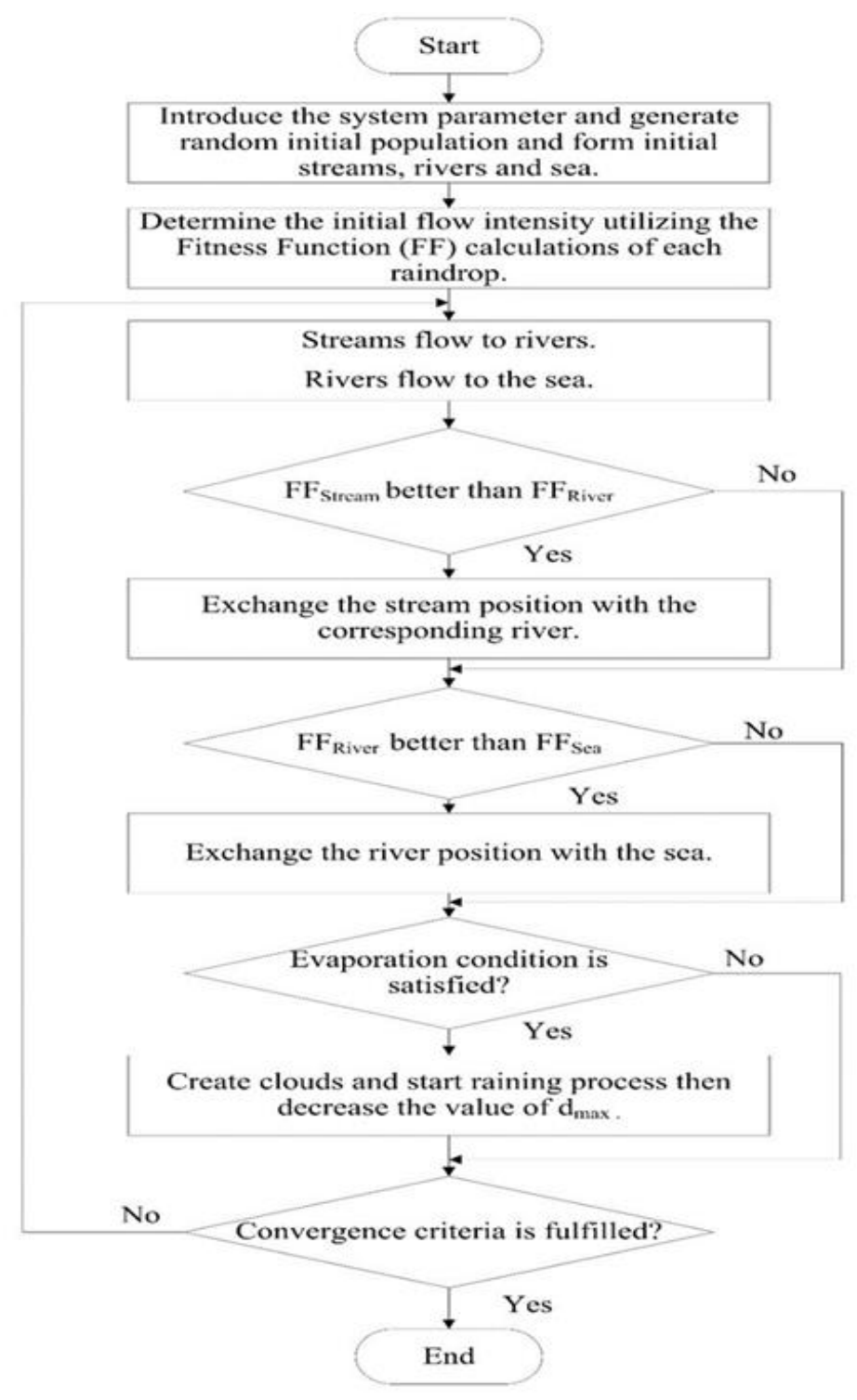

Figure 4. The flowchart for the Water Cycle Optimization Technique (WCOT) (Eskandar et al. 2012). 


\section{Results and Discussion}

In this section, the comparison between the MILP, GA, and WCOA has been investigated to ensure the optimum charging ranking of $15 \mathrm{EVs}$ entering the station at the same time as the procedures in Fig.5. The proposed scheduling is depending on selecting the charging methodology, optimizing the charging capacity within an acceptable range from $85 \%$ to $100 \%$ of the total capacity of the battery, and minimizing the total charging time in the EVS.

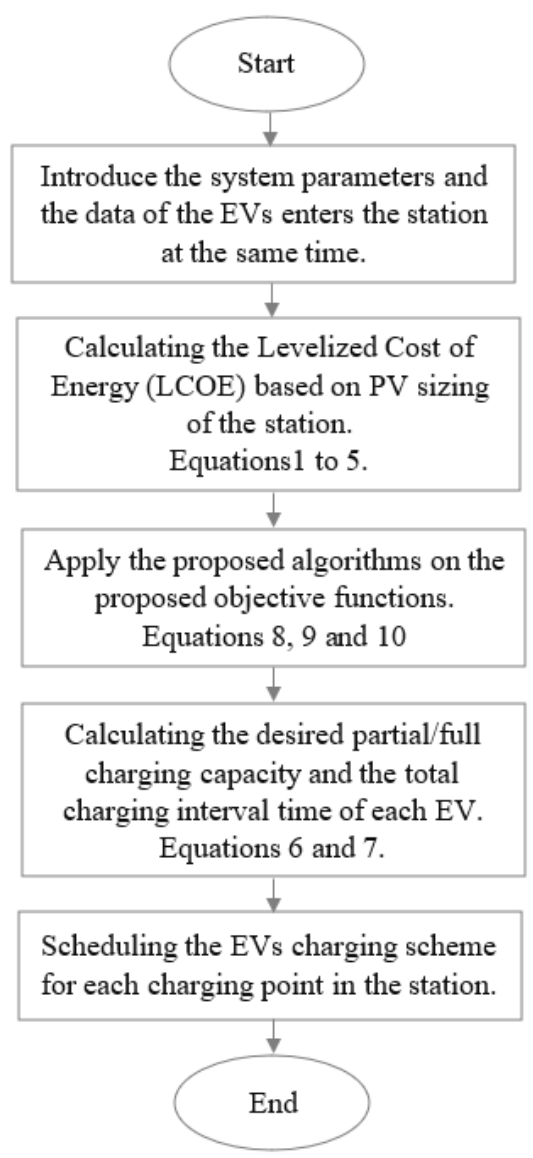

Figure 5. The illustrative flowchart of the proposed decision-making criteria

The results of the proposed optimizing algorithms are depending on the charging methodology, coefficient of partial charging capacity, and the density coefficient of the station. The scheduling of the EVs in the charging station has been presented in Fig. 6 where the methodology used in charging the EV, the partial charging capacity, and the corresponding charging point for each EV have been introduced. 
It is observed that the total charging time of the proposed 15 EVs using MILP, GA, and WCOA is $51.3 \mathrm{mins}, 48.6 \mathrm{mins}$, and $44.5 \mathrm{mins}$, respectively. MILP is considered as the lowest efficient algorithm used to solve such an optimization problem. This shows high consistency with the previously reported data in the literature, concerning the inapplicability of linear programming techniques in EV decision-making algorithms, the impossibility of considering nonlinear effects, and the risk of the high dimensionality of the problems in comparison with other advanced optimization techniques (Moghaddam et al. 2017; Ki et al. 2018; Urbanucci 2018). This is quantitatively attributed to the fact that $40 \%$ of the EV has chosen DC-CCCV as a charging alternative in MILP, while $60 \%$ and $73 \%$ of EVs have chosen DC-CCCV in WCOA and GA, respectively. Principally, this observation should inflate GA and WCOA total charging time in comparison with MLIP, as the average charging time for DC-CCCV is significantly higher than that of DC-MSCC. However, the impact of both the queuing delay and the partial chagrining capacity coefficient influences the performance of both GA and WCOA over the MILP. It is easily observed that the metaheuristic algorithms recorded dynamic partial charging coefficient, depending on the EV initial parameters, while a maximum partial charging coefficient is always observed in the MILP model.

On the other hand, the observed results, while considering the efficiency of the WCOA against GA and MILP, emphasizes the attractiveness of the suggested model in handling the optimization problem under study. The obtained results show that the proposed algorithm offers better solutions than other optimizers considered in this work. Although, WCOA proved its effectiveness in different challenging benchmark optimization problems with lots of local optimization points investigated in different research areas as stated in (Dizangian and Hooshyari 2017; Makeen et al. 2018; Eskandar et al. 2012) in addition to its efficiency in terms of the number of function evaluations (computational cost) for the considered nonconvex problem.

Besides, the WCOA ensured a speed computational time response where only 2.67 secs have been preordained to ensure the optimum combination of the EV's charging interval time till leaving the station, the targeted partial/full charging capacity, and the appropriate charging methodology. However, the GA and MILP spent 2.9 and 3.168 secs respectively to find the optimum combination from their perspective. 


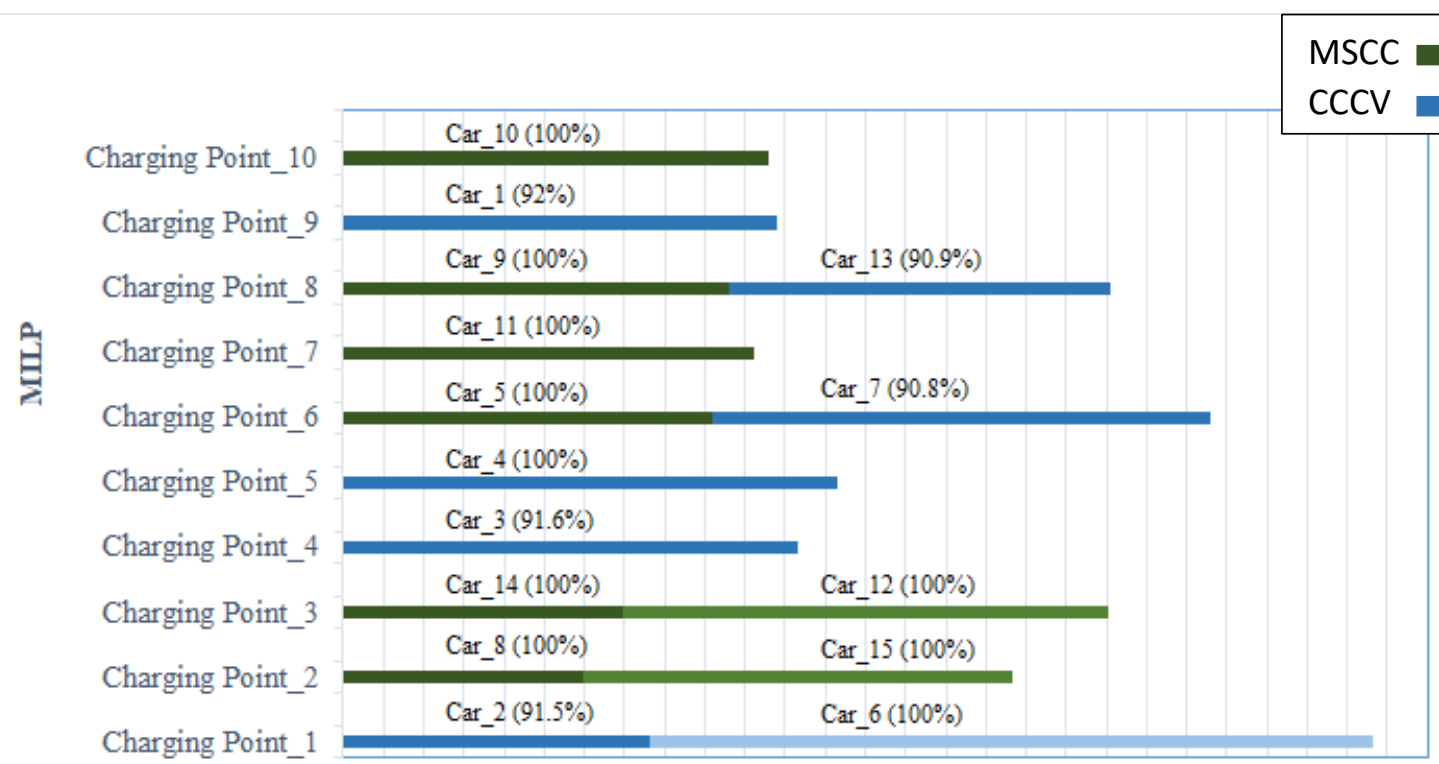

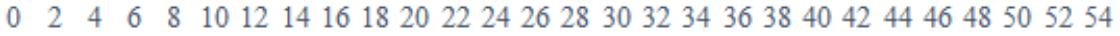
Charging time (min)

(a)

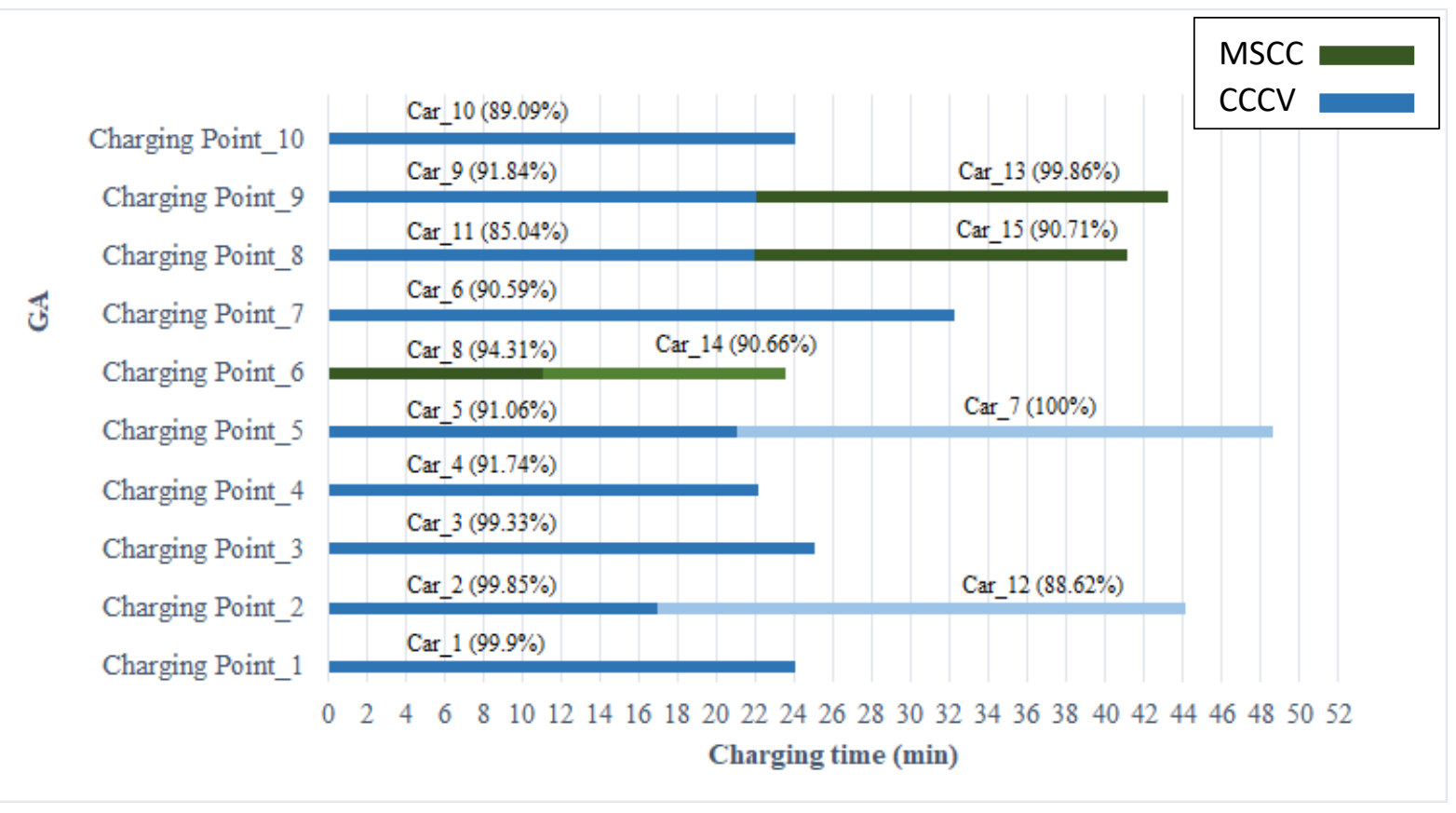

(b) 


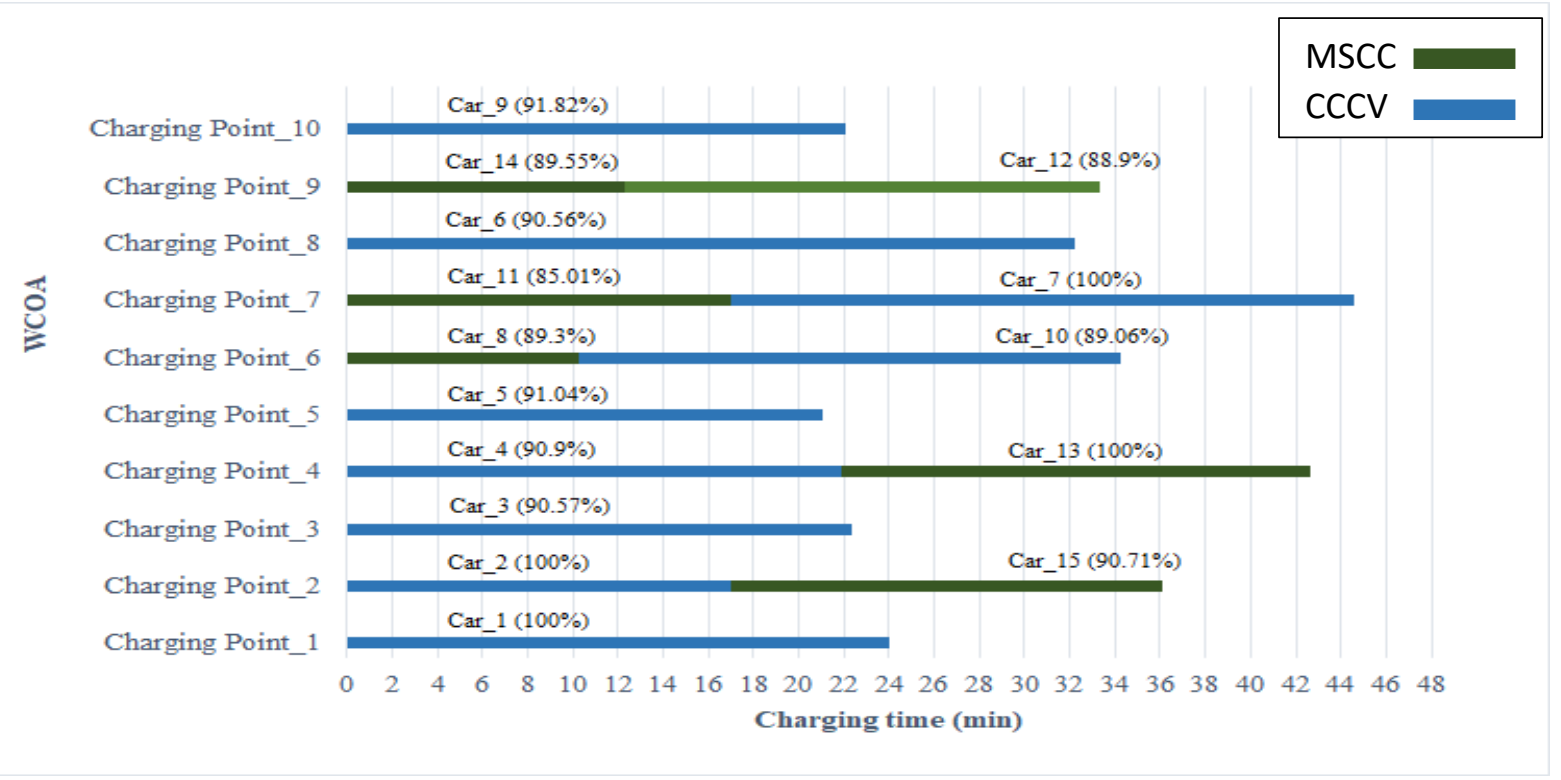

(c)

Figure 6. EVS smart charging scheme of Mixed Integer Linear Programming (MILP), Genetic Algorithm (GA), and Water Cycle Optimization Technique (WCOT) respectively.

\section{Conclusion}

Green and sustainable electrification for the automation industry is considered as a proactive model to minimize global pollution. However, providing optimum charging time in the centralized stations is still missing. In this paper, we highlighted the utilization of an integrated Photovoltaic/grid scenario to provide sustainable energy for an electric vehicle station. A decisionmaking criterion based on a multi-objective function has been presented to schedule the EVs charging scheme seeking minimum total charging interval time of the centralized station, selecting the appropriate charging methodology (CCCV or MSCC), and the targeted partial/full charging capacity for each EV that satisfies long lifespan of the battery. Three optimizers have been investigated by utilizing the mixed-integer linear programming and metaheuristic algorithms (GA and WCOA). It was observed that the metaheuristic algorithms, specifically the water cycle optimization algorithm, have reported the minimum total charging time with a $13 \%$ reduction in comparison with the MILP algorithm. Additionally, metaheuristic algorithms have shown an interesting capability in optimizing the partial charging capacity coefficient in terms of the initial EV parameters. Finally, the WCOA has glowed up as a potential optimizer in terms of the 
computational cost and time response against other introduced models, while recording the minimum charging time.

The current optimization model has been tested on a limited number of EVs due to the manually structured databased used for EVs, however in order to examine the model performance with increasing the number of EVs, a more advanced artificial model with a database management system can be used. Machine learning, as well as deep learning, may be utilized in optimizing such EV optimization problem. the authors consider such optimizer as the future extension of the current work. 


\section{References}

Abdel-aal, H Mantawy. 2012. "Genetic Algorithms Application to Electric Power Systems." Review of. Genetic Algorithms in Applications 22:91.

Ahmad, Furkan, Mohammad Saad Alam, Samir M Shariff, and Mahesh Krishnamurthy. 2019. "A cost-efficient approach to EV charging station integrated community microgrid: A case study of Indian power market." Review of. IEEE Transactions on Transportation Electrification 5 (1):200-14.

Amjad, Muhammad, Ayaz Ahmad, Mubashir Husain Rehmani, and Tariq Umer. 2018. "A review of EVs charging: From the perspective of energy optimization, optimization approaches, and charging techniques." Review of. Transportation Research Part D: Transport and Environment 62:386-417.

Chen, Tao, Bowen Zhang, Hajir Pourbabak, Abdollah Kavousi-Fard, and Wencong Su. 2016. "Optimal routing and charging of an electric vehicle fleet for high-efficiency dynamic transit systems." Review of. IEEE Transactions on Smart Grid 9 (4):3563-72.

Cheng, Xiao, Jinma Sheng, Xiuting Rong, Hui Zhang, Lei Feng, and Sujie Shao. 2020. "Ordered Electric Vehicles Charging Scheduling Algorithm Based on Bidding in Residential Area." Review of. Information 11 (1):49.

Dasgupta, Dipankar, and Zbigniew Michalewicz. 2013. Evolutionary algorithms in engineering applications: Springer Science \& Business Media.

Dizangian, Babak, and Ali Hooshyari. 2017. "Comparing the particle swarm, whale, water cycle, and cuckoo search algorithms in optimization of unconstrained problems." Review of.

Domínguez-Navarro, JA, R Dufo-López, JM Yusta-Loyo, JS Artal-Sevil, and JL Bernal-Agustín. 2019. "Design of an electric vehicle fast-charging station with integration of renewable energy and storage systems." Review of. International Journal of Electrical Power E Energy Systems 105:46-58.

Eskandar, Hadi, Ali Sadollah, Ardeshir Bahreininejad, and Mohd Hamdi. 2012. "Water cycle algorithm-A novel metaheuristic optimization method for solving constrained engineering optimization problems." Review of. Computers \& Structures 110:151-66.

Gupta, Pradeep Kumar, Rubi Rana, and Sukumar Mishra. 2020. Multi Priority-Queuing algorithm for Real Time Charge Scheduling of Electric Vehicles on Highways based on Time allotment. Paper presented at the 2020 IEEE International Conference on Power Electronics, Smart Grid and Renewable Energy (PESGRE2020).

https://ev-database.org/car/1178/SEAT-el-Born\#charge-table. 2020. "Electric Vehicles battaries database ", Accessed 04.

Huang, Yantao, and Kara M Kockelman. 2020. "Electric vehicle charging station locations: Elastic demand, station congestion, and network equilibrium." Review of. Transportation Research Part D: Transport and Environment 78:102179.

Ki, Youngmin, Byung-In Kim, Young Myoung Ko, Hyemoon Jeong, and Jeongin Koo. 2018. "Charging scheduling problem of an M-to-N electric vehicle charger." Review of. Applied Mathematical Modelling 64:603-14.

Korkas, Christos D, Simone Baldi, Shuai Yuan, and Elias B Kosmatopoulos. 2017. "An adaptive learning-based approach for nearly optimal dynamic charging of electric vehicle fleets." Review of. IEEE Transactions on Intelligent Transportation Systems 19 (7):2066-75.

Liang, Yanchang, Zhaohao Ding, Tao Ding, and Wei-Jen Lee. 2020. "Mobility-Aware Charging Scheduling for Shared On-Demand Electric Vehicle Fleet Using Deep Reinforcement Learning." Review of. IEEE Transactions on Smart Grid.

Makeen, P., H. Ghali, and S. Memon. 2020a. "Controllable Electric Vehicle Fast Charging Approach Based on MultiStage Charging Current Methodology." Review of. 2020 IEEE International Conference on Power and Energy (PECon):398-403.

Makeen, Peter, Hani Ghali, and Saim Memon. 2020b. "Experimental and theoretical analysis of the fast charging polymer lithium-ion battery based on Cuckoo Optimization Algorithm (COA)." Review of. Ieee Access.

Makeen, Peter, RA Swief, TS Abdel-Salam, and Noha H El-Amary. 2018. "Smart hybrid micro-grid integration for optimal power sharing-based water cycle optimization technique." Review of. Energies 11 (5):1083.

Mehrjerdi, Hasan, and Reza Hemmati. 2020. "Stochastic model for electric vehicle charging station integrated with wind energy." Review of. Sustainable Energy Technologies and Assessments 37:100577.

Moghaddam, Zeinab, Iftekhar Ahmad, Daryoush Habibi, and Quoc Viet Phung. 2017. "Smart charging strategy for electric vehicle charging stations." Review of. IEEE Transactions on Transportation Electrification 4 (1):76-88. 
Rabiee, Abdorreza, Ali Ghiasian, and Moslem Amiri Chermahini. 2018. "Long term profit maximization strategy for charging scheduling of electric vehicle charging station." Review of. IET Generation, Transmission E Distribution 12 (18):4134-41.

Reddi, K, M Mintz, A Elgowainy, and E Sutherland. 2016. "Building a hydrogen infrastructure in the United States." In Compendium of hydrogen energy, 293-319. Elsevier.

Said, Dhaou, and Hussein T Mouftah. 2019. "A novel electric vehicles charging/discharging management protocol based on queuing model." Review of. IEEE Transactions on Intelligent Vehicles 5 (1):100-11.

Shi, Jiancheng, Xiaolong Dong, Tianjie Zhao, Yang Du, Hao Liu, Zhenzhan Wang, Di Zhu, Dabin Ji, Chuan Xiong, and Lingmei Jiang. 2016. The water cycle observation mission (WCOM): Overview. Paper presented at the 2016 IEEE International Geoscience and Remote Sensing Symposium (IGARSS).

Trespalacios, Francisco, and Ignacio E Grossmann. 2014. "Review of mixed-integer nonlinear and generalized disjunctive programming methods." Review of. Chemie Ingenieur Technik 86 (7):991-1012.

Ucer, Emin, Işıl Koyuncu, Mithat C Kisacikoglu, Mesut Yavuz, Andrew Meintz, and Clément Rames. 2019. "Modeling and analysis of a fast charging station and evaluation of service quality for electric vehicles." Review of. IEEE Transactions on Transportation Electrification 5 (1):215-25.

Urbanucci, Luca. 2018. "Limits and potentials of Mixed Integer Linear Programming methods for optimization of polygeneration energy systems." Review of. Energy Procedia 148:1199-205.

Vorobjovs, Maksims, Kristina Berzma, and Anastasija Zirovecka. 2018. Electric Vehicle Charging Station Load Analyzing Based on Monte-Carlo Method. Paper presented at the 2018 20th European Conference on Power Electronics and Applications (EPE'18 ECCE Europe).

Wang, Shuoyao, Suzhi Bi, Ying-Jun Angela Zhang, and Jianwei Huang. 2018. "Electrical vehicle charging station profit maximization: Admission, pricing, and online scheduling." Review of. IEEE Transactions on Sustainable Energy 9 (4):1722-31.

Wu, Hao, Grantham Kwok Hung Pang, King Lun Choy, and Hoi Yan Lam. 2017. "An optimization model for electric vehicle battery charging at a battery swapping station." Review of. IEEE Transactions on Vehicular Technology 67 (2):881-95.

Zhang, Tian, Wei Chen, Zhu Han, and Zhigang Cao. 2013. "Charging scheduling of electric vehicles with local renewable energy under uncertain electric vehicle arrival and grid power price." Review of. IEEE Transactions on Vehicular Technology 63 (6):2600-12.

Zhang, Yongmin, Pengcheng You, and Lin Cai. 2018. "Optimal charging scheduling by pricing for EV charging station with dual charging modes." Review of. IEEE Transactions on Intelligent Transportation Systems 20 (9):3386-96. 